\section{Next-gen polyamine attack}

\section{By Brian Moy, Staff Writer}

The development of eflornithine monotherapy to treat cancer suffered a significant setback in 2004 when Ilex Oncology Inc., since acquired by Genzyme Corp., reported that the polyamine biosynthesis inhibitor failed to prevent recurrence of bladder cancer in a Phase III trial. Although polyamine metabolism is frequently dysregulated in cancer cells, eflornithine monotherapy was stymied by two issues: toxicity at high doses and the ability of cells to import polyamines from external sources and circumvent the action of polyamine biosynthesis inhibitors.

Aminex Therapeutics Inc. has developed a series of lipophilic lysine-spermine conjugates that may address both problems. The conjugates, which act as polyamine transport inhibitors, were used in combination with eflornithine (DFMO) in a two-pronged attack to deprive cancer cells of the polyamines they need for continued cell growth and proliferation.

The company used a mouse model of squamous cell carcinoma that overexpressed ornithine decarboxylase (ODC) - the rate-limiting enzyme in polyamine biosynthesis that is eflornithine's target. As reported in the Journal of Medicinal Chemistry, the combination of DFMO and a lysine-spermine conjugate resulted in substantially fewer visible tumors six weeks after treatment compared with that seen using either agent alone. ${ }^{1}$

In 8 of 17 mice receiving the combination for 4 weeks, no tumors were detected following 6 weeks off treatment. By contrast, tumors regrew in all mice receiving the polyamine transport inhibitor alone and in 11 of 12 mice receiving DFMO alone.

"The potential of the polyamine transport inhibitors and the resulting two-drug combination therapy to become a next-generation chemotherapeutic regimen is very promising, as demonstrated by the positive results in the murine model," said Mark Burns, lead author of the JMC paper and CEO of Aminex.

Aminex is developing polyamine-based therapies to treat epithelial-based cancers, including squamous cell carcinoma of the head and neck (SCCHN), melanoma and colon cancer. The company hopes to submit an IND by $1 \mathrm{Q} 10$ for a polyamine transport inhibitor plus DFMO to treat SCCHN.

Burns characterized the lysine-spermine conjugates while he was working at MediQuest Therapeutics Inc. Last year, he left the company and formed Aminex, which holds an exclusive license from MediQuest to patents covering the compounds.

\section{Toxic limitations}

The next steps for the combination therapy involve determining potential toxicity and finding an optimal dosing regimen. A key issue is that DFMO monotherapy has been associated with gastrointestinal and hematological side effects and loss of hearing at high doses. $^{2}$

Recent studies have shown that lower doses of DFMO administered over long periods of time can still be effective in lowering polyamines without producing toxic side effects. ${ }^{3}$ Thus, Burns said that detailed studies will be needed to determine the best ratio of the individual components of the combination therapy.

"The efficacy of polyamine transport inhibitors looks very promising in experimental models. However, the major question is whether or not there will be toxicity when the compounds are administered to humans," said Eugene Gerner, director of the Arizona Cancer Center's gastrointestinal cancer program and a professor of cell biology, anatomy, biochemistry and molecular biophysics at the University of Arizona.

He added: "It will be important to conduct Phase I safety and toxicity studies in patients with advanced cancers, and then, in the context of those studies, there needs to be some indication of benefit."

Laurence Marton, CSO at cancer company Progen Pharmaceuticals Ltd., agreed. "The next step is to look more formally at toxicity and see how the animal as a whole is affected when treated with the transport inhibitors alone, with DFMO alone and then with combination therapy."

\section{Other polyamine approaches}

Aminex is not the only company developing cancer therapies that interfere with the polyamine pathway.

Progen is developing polyamine analogs that are transported into cells and are incorporated into intracellular polyamine binding sites. Once bound, the analogs have altered function compared with natural polyamines, modulating polyamine biosynthetic pathways and altering cell growth by inducing apoptosis and cell-cycle arrest.

The company's PG11047 polyamine analog is in Phase I testing to treat advanced cancer as monotherapy and in combination with marketed drugs.

Cancer Prevention Pharmaceuticals LLC, a company spun out of the University of Arizona and the University of California, Irvine, is developing compounds to prevent cancer in people with increased risk for disease. The company hopes to submit an NDA in 2010 for a combination of DFMO and sulindac to prevent colorectal cancer. Sulindac is a generic NSAID.

Gerner, who is Cancer Prevention's CSO, noted that the company's approach is aimed at inhibiting synthesis and promoting export of polyamines, whereas the approach reported in the JMC paper seeks to inhibit synthesis and block polyamine transport and uptake.

Moy, B. SciBX 2(14); doi:10.1038/scibx.2009.562

Published online April 9, 2009 


\section{TARGETS \& MECHANISMS}

\section{REFERENCES}

1. Burns, M. et al. J. Med. Chem.; published online March 12, 2009; doi:10.1021/jm801580w

Contact: Mark R. Burns, Aminex Therapeutics Inc., Seattle, Wash.

e-mail: markburns@aminextherapeutics.com

2. Meyskens, F. \& Gerner, E. Clin. Cancer Res. 5, 945-951 (1999)

3. Meyskens, F. et al. J. Natl. Cancer Inst. 90, 1212-1218 (1998)

\author{
COMPANIES AND INSTITUTIONS MENTIONED \\ Aminex Therapeutics Inc., Seattle, Wash. \\ Cancer Prevention Pharmaceuticals LLC, Tucson, Ariz. \\ Genzyme Corp. (NASDAQ:GENZ), Cambridge, Mass. \\ MediQuest Therapeutics Inc., Bothell, Wash. \\ Progen Pharmaceuticals Ltd. (ASX:PGL; NASDAQ:PGLA), \\ Brisbane, Australia \\ University of Arizona, Tucson, Ariz. \\ University of California, Irvine, Calif.
}

